einstein

Official Publication of the Instituto Israelita

de Ensino e Pesquisa Albert Einstein

ISSN: 1679-4508 | e-ISSN: 2317-6385

\title{
COVID-19 and the gastrointestinal tract: what do we already know?
}

\section{COVID-19 e o trato gastrintestinal: o que já sabemos?}

\author{
Joana Ferro Machado de Almeida', Ethel Zimberg Chehter ${ }^{1}$ \\ ${ }^{1}$ Faculdade de Medicina do ABC, Santo André, SP, Brazil.
}

DOI: 10.31744/einstein_journal/2020RW5909

\section{ABSTRACT}

The new coronavirus disease pandemic is defining 2020, with almost 17.5 million infected individuals and 700 thousand deaths up to beginning of August. It is caused by SARS-CoV-2 and the transmission is through the respiratory tract. Those infected may be asymptomatic, present typical symptoms (fever, dry cough and dyspnea), gastrointestinal symptoms (diarrhea, nausea, vomiting and abdominal pain) and viral RNA in stools. The objective of this work was to review the literature related to the prevalence of gastrointestinal symptoms, and to check the possibility of fecal-oral transmission. We searched PubMed ${ }^{\circledR}$ database on COVID-19 and gastrointestinal tract and selected articles using the PRISMA method. We eliminated articles based on titles and abstracts, small number of patients and the mechanism of infection, leaving 14 studies. Comorbidities and laboratory alterations (elevation of hepatic aminotransferases and bilirubin) were related to worsening of the disease. The prevalence of gastrointestinal symptoms ranged from $6.8 \%$ to $61.3 \%$, including diarrhea ( $8.14 \%$ to $33.7 \%$ ), nausea/vomiting ( $1.53 \%$ to $26.4 \%$ ), anorexia (12.1\% to $40.0 \%$ ) and abdominal pain (0\% to $14.5 \%)$. The presence of viral RNA in stools was rarely tested, but positive in $0 \%$ to $48.1 \%$. The gastrointestinal tract is affected by COVID-19, causing specific symptoms, laboratory alterations and viral presence in the feces. However, the results of prevalence and possibility of fecal-oral transmission were varied, requiring further studies for more assertive conclusions. It is important that healthcare professionals draw attention to this fact, since these changes can help make diagnosis and initiate early treatment.

How to cite this article:

Almeida JF, Chehter EZ. COVID-19 and the gastrointestinal tract: what do we already know? einstein (São Paulo). 2020;18:eRW5909. http://dx.doi.org/10.31744/ einstein journal/2020RW5909

\section{Corresponding author: \\ Joana Ferro Machado de Almeida Centro Universitário Saúde ABC - \\ Departamento Gastroclínica Anexo 2 Avenida Príncipe de Gales, 821 - Portaria 1 Zip code: 09060-650 - Santo André, SP, Brazil Phone: (55 11) 4993-5400 \\ E-mail: joana.ferromalmeida@gmail.com}

\section{Received on:}

June 8, 2020

\section{Accepted on:}

Aug 27, 2020

\section{Copyright 2020}

\section{(cc) Br}

This content is licensed

under a Creative Commons

Attribution 4.0 International License.
Keywords: SARS-CoV-2; Betacoronavirus; Coronavirus infections; COVID-19; Gastrointestinal tract; Gastrointestinal diseases/etiology

\section{RESUMO}

Com quase 17,5 milhões de infectados e 700 mil mortos até o início de agosto no mundo, a pandemia do novo coronavírus está marcando o ano de 2020. 0 agente causador da doença é 0 vírus SARS-CoV-2, e a transmissão é por via respiratória. Os infectados podem ser assintomáticos, apresentar sintomas típicos (febre, tosse seca e dispneia), sintomas gastrintestinais (diarreia, náusea, vômito e dor abdominal) e RNA viral nas fezes. 0 objetivo deste trabalho foi revisar a literatura relacionada com a prevalência dos sintomas gastrintestinais, e verificar se é possível a transmissão fecal-oral da doença. Fizemos uma pesquisa na base de dados PubMed ${ }^{\circledR}$ sobre a COVID-19 e o trato gastrintestinal, selecionando artigos pelo método PRISMA. Eliminamos artigos com base em títulos e resumos, quantidade pequena de pacientes e sobre mecanismo de infecção, restando 14 estudos. Comorbidades e alterações laboratoriais (elevação de aminotransferases hepáticas e bilirrubina) foram relacionadas com piora da doença. A prevalência de sintomas gastrintestinais variou entre $6,8 \%$ e $61,3 \%$, sendo eles diarreia $(8,14 \%$ a 33,7\%), náusea/vômito $(1,53 \%$ a $26,4 \%)$, anorexia $(12,1 \%$ a $40,0 \%)$ e dor abdominal ( $0 \%$ a $14,5 \%)$. A presença do RNA viral foi pouco testada, mas foi positiva entre $0 \%$ a $48,1 \%$. 0 trato gastrintestinal é muito acometido pela COVID-19, provocando sintomas específicos, alterações laboratoriais e presença viral nas fezes. Contudo, os resultados de prevalência e a possibilidade de transmissão fecal-oral foram 
variados, necessitando de estudos maiores para conclusões mais assertivas. É importante a atenção de profissionais da saúde a isso, visto que essas alterações podem ajudar no diagnóstico e a iniciar tratamento precoce.

Descritores: SARS-CoV-2; Betacoronavírus; Infecções por coronavírus; COVID-19; Trato gastrointestinal; Doenças gastrointestinais/etiologia

\section{INTRODUCTION}

In December 2019, the first cases of patients infected with the new coronavirus were identified in Wuhan, China. ${ }^{(1)}$ The year 2020 will be remembered by the 2019 coronavirus pandemic (COVID-19), causing a great impact on public health and economy of many countries. On August 1 $1^{\text {st }}, 2020$, according to the World Health Organization (WHO) website, there were 17,396,943 cumulative cases and 675,060 deaths worldwide. The country with the highest number of infected individuals and deaths is the United States, with 4,456,389 and 151,265 , respectively. In Brazil, these figures were 2,610,102 cases and 91,263 deaths. ${ }^{(2)}$

The virus, called severe acute respiratory syndrome coronavirus 2 (SARS-CoV-2), is the seventh coronavirus known to infect humans. The others are the acute severe respiratory syndrome coronavirus (SARS-CoV), with an epidemic in 2003, and the Middle Eastern respiratory syndrome coronavirus (MERS-CoV), with an epidemic in the Middle East, in 2012, and both cause severe disease; and HKU1, NL63, OC43, and 229E, which cause mild symptoms. SARS-CoV-2 is an encapsulated RNA virus of the order Nidovirales, family Coronaviridae, subfamily Coronavirinae, genus Beta. ${ }^{(3)}$ SARS-CoV-2 probably originated in bats and is transmitted to humans through another animal. ${ }^{(4)}$ The main transmission route is respiratory, through contact with droplets, aerosols, and contaminated surfaces. ${ }^{(5)}$

Typical symptoms of the disease are fever, dry cough, dyspnea, headache, anosmia, dysgeusia, and pneumonia, but it can also be asymptomatic. It may cause progressive respiratory failure through alveolar injury, which may lead to death. ${ }^{(1,6)}$ However, several studies indicate that patients may present symptoms related to the gastrointestinal tract (GIT), with the presence of the virus in epithelial cells of the GIT and its RNA in feces. Based on this, a possible oralfecal transmission of the disease is suggested. ${ }^{(6,7)}$ The main gastrointestinal symptoms are diarrhea, nausea, vomiting, and abdominal pain. ${ }^{(8)}$

The SARS-CoV-2 virus uses the angiotensin 2 converting enzyme (ACE2) as a receptor for entrance into cells. It mainly infects lung cells, but may have gastrointestinal involvement, which also has ACE2 receptors at the brush border of the intestinal mucosa. ${ }^{(6)}$ In addition, the virus is released at the apical part of the lung cells. Dragged by mucociliary movement, it can gain the GIT. These ACE2 receptors operate diminishing the action of the renin-angiotensinaldosterone system by metabolizing angiotensin 2 . At high serum levels, angiotensin 2 has effects on cardiovascular, renal, and respiratory systems, and may cause chronic hypertension and renal and respiratory failure. The SARS-CoV-2 virus causes downregulation and blockage of these ACE2 receptors, which explains the clinical picture of pulmonary failure and adult respiratory distress syndrome in some infected patients..$^{(9)}$

The viral surface has S (Spike) glycoproteins, which need to be separated into S1 and S2 domains, so that the virus can adhere to the cell membrane. Furin and transmembrane serine protease (TMPRSS2) are substances that allow this separation. Thus, S1 binds to the ACE2 receptor and S2 to the cell membrane, allowing the virus to enter the cell through endocytosis. Furin and TMPRSS2 are enzymes present in the cells of the small intestine; the former also acts in the activation of toxins of some microorganisms. ${ }^{(7,9)}$

ACE2 of the intestinal mucosa is associated with the amino acid carrier, $\mathrm{B}^{0} \mathrm{AT} 1$, and regulates the intestinal flora. This occurs because this transporter allows the absorption of tryptophan, which stimulates the mTOR path to produce antimicrobial peptides. Thus, the SARS-CoV-2 infection changes the amount, and blocks the ACE2 receptors in the brush edge, causing tryptophan deficiency and lower production of antimicrobial peptides, which in turn can cause changes in the intestinal microbioma and inflammation. ${ }^{(7,9)}$

Patients with COVID-19 present with a great inflammatory reaction caused by the so-called cytokine storm, which can be originated or potentiated by the GIT. The small intestine has the largest amount of lymphoid tissue in the body, with Peyer patches, mesenteric lymph nodes, and lymphoid follicles along the intestine. In the mucosa and below the lamina propria, the intestines also have a large population of activated $\mathrm{T}$ cells, plasma cells, mast cells, dendritic cells, and macrophages. In the scenario of an infection such as COVID-19, there is an exaggerated release of cytokines, which promote the recruitment of several other cells, causing a great inflammatory process. ${ }^{(9,10)}$ Studies point out that interferon and influenza virus infection (interferon pathway inducer) may be related to increased ACE2 transcription. ${ }^{(11)}$ Smokers and patients with chronic obstructive pulmonary disease (COPD) also have a higher expression of ACE2 receptors. ${ }^{(12)}$ In addition, 
diabetes and hypertension have been associated with polymorphisms of the ACE2 gene. ${ }^{(7)}$

Drugs used in the treatment of hypertension, such as ACE inhibitors (ACEi) and angiotensin receptor blockers (ARB), reduce inflammation by decreasing cytokines. In addition, they may increase the expression of ACE2. ${ }^{(13)}$ However, the use of ACEi and ARB has not been associated with a worse clinical evolution in patients with COVID-19. Thus, maintenance of the use of these drugs in hypertensive patients is recommended. ${ }^{(14,15)}$

While the SARS-CoV and SARS-CoV-2 viruses use ACE2 as a mediator to enter the cells, MERS-CoV uses dipeptidyl-peptidase 4 (DPP4). ${ }^{(7)}$ Because they are viruses from the same family, some authors have studied whether DPP4 is also involved in the infection mechanism of the new disease. According to Vankadari et al., the S1 domain of the SARS-CoV-2 membrane glycoprotein, besides interacting with ACE2, the main gateway for the new virus may have some relation with DPP4. ${ }^{(16)}$ However, the study by Tai et al., found that the virus did not bind to $293 \mathrm{~T}$ cells that expressed only DPP4. This connection only occurred in $293 \mathrm{~T}$ cells with ACE2.(17)

Several articles on GIT-related symptoms of COVID-19 and its possible oral-fecal transmission have been published, with divergent results.

The objective of this study was to review the literature related to the prevalence of gastrointestinal symptoms, and to verify the possibility of fecal-oral transmission of the disease.

\section{METHODS}

The database chosen for this work was PubMed ${ }^{\circledR}$. On May 5, 2020, we searched for articles with the words "COVID-19" or "SARS-CoV-2" and "GIT" or "gastrointestinal" or "enteric." A total of 179 articles resulted from this search. The inclusion criteria for the studies in this review were the fulfillment of eligibility criteria (articles addressing SARS-CoV-2 infection and GIT symptoms), articles in English, and samples with more than 50 patients. Exclusion criteria were study design of case reports, reviews on the infection mechanism and virus, GIT-related procedure recommendation manuals in infected patients, articles in languages other than English, and less than 50 patients evaluated. Figure 1 shows the diagram Preferred Reporting Items for Systematic Reviews and Meta-Analyses (PRISMA) performed based on reading titles and abstracts; 161 articles were eliminated, leaving 18 articles. One last article was added later. Of these 19 articles, four were reviews about the virus and its mechanism of infection, which were disregarded. Finally, a study including only ten patients was eliminated. With this, the review was carried out with 14 articles, in that four were metaanalyses, one systematic review, and nine retrospective studies. The analysis of the articles was initially made by the author, but the final choice also included a senior reviewer. Due to the small number of large studies related to this subject, and the importance and urgency of the topic, several variations of study designs were included. Information on country of origin, mean age, different comorbidities, typical symptoms (fever, cough, and dyspnea, among others), gastrointestinal symptoms (diarrhea, nausea, vomiting, and abdominal pain), and the presence of viral RNA in feces, when cited, were included in this study for analysis.

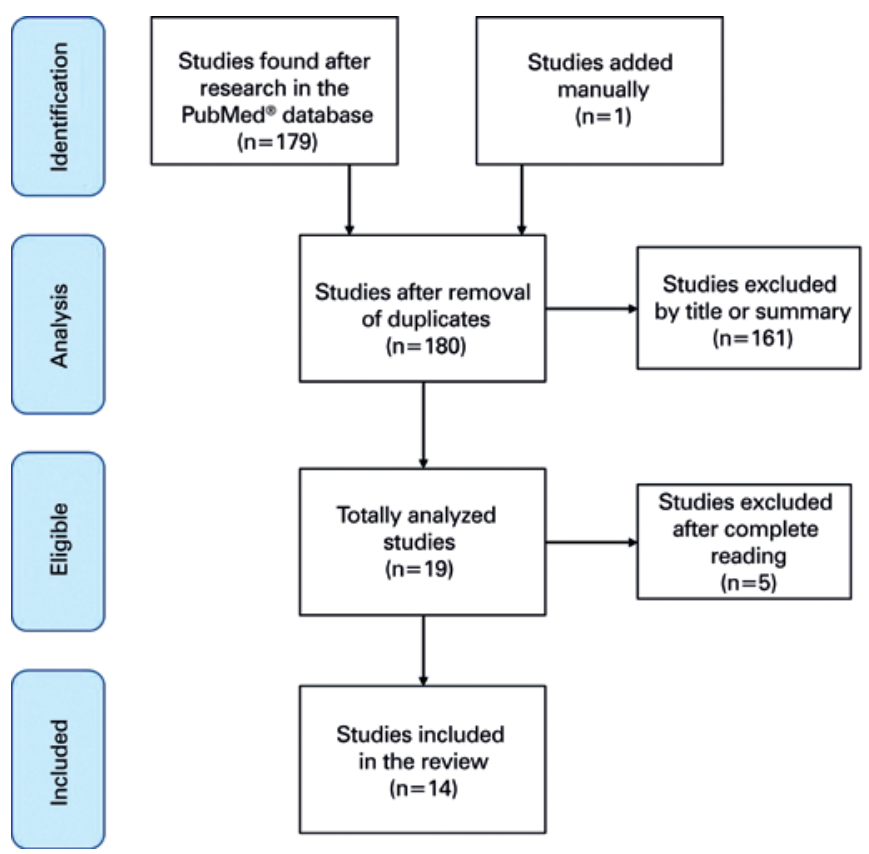

Figure 1. Preferred Reporting Items for Systematic Reviews and Meta-Analyses Diagram (PRISMA)

\section{| RESULTS}

As to retrospective studies, three were done only with patients from the United States and six only from China. The systematic review included several studies carried out in China. As to the meta-analyses, two of them included studies done only in China and two evaluated various countries. Tables 1 to 3 present general data, typical symptoms, and gastrointestinal symptoms of patients with COVID-19 of the retrospective studies, respectively. Table 4 displays the symptoms of patients with the disease in the meta-analyses. 
Table 1. General data of patients with COVID-19 of the nine retrospective studies

\begin{tabular}{|c|c|c|c|c|c|c|c|c|c|c|c|c|}
\hline & & \multicolumn{3}{|c|}{ Jin et al. ${ }^{(18)}$} & \multicolumn{4}{|c|}{ Lin et al. ${ }^{(19)}$} & \multicolumn{4}{|c|}{ Pan et al. ${ }^{(20)}$} \\
\hline & & $\begin{array}{c}\text { With GI } \\
\text { symptoms }\end{array}$ & $\begin{array}{l}\text { Without GI } \\
\text { symptoms }\end{array}$ & p value & Total & $\begin{array}{c}\text { With GI } \\
\text { symptoms }\end{array}$ & $\begin{array}{l}\text { Without GI } \\
\text { symptoms }\end{array}$ & p value & Total & $\begin{array}{c}\text { With GI } \\
\text { symptoms }\end{array}$ & $\begin{array}{l}\text { Without GI } \\
\text { symptoms }\end{array}$ & p value \\
\hline \multicolumn{2}{|l|}{ Patients } & 74 & 577 & & 95 & 58 & 37 & & 204 & 103 & 101 & \\
\hline \multicolumn{2}{|l|}{ Age } & $46.14 \pm 14.19$ & $45.09 \pm 14.45$ & 0.559 & $43.3 \pm 18.3$ & $48.0 \pm 17.1$ & $41.1 \pm 19.5$ & 0.073 & $52.91 \pm 15.98$ & $52.21 \pm 15.92$ & $53.61 \pm 16.10$ & 0.533 \\
\hline \multicolumn{2}{|l|}{ Sex } & $\begin{array}{l}M: 37 / 74(50.0) \\
W: 37 / 74(50.0)\end{array}$ & $\begin{array}{l}\text { M: 294/577 (51) } \\
\text { W: 283/577 (49) }\end{array}$ & M: 0.902 & $\begin{array}{l}\text { M: 45/95 (47.4) } \\
\text { W: 50/95 (52.6) }\end{array}$ & $\begin{array}{l}M: 27 / 58(46.6) \\
W: 31 / 58(53.4)\end{array}$ & $\begin{array}{l}\text { M: 18/37 (48.6) } \\
\text { W: 19/37 (51.4) }\end{array}$ & W: 0.84 & $\begin{array}{l}\text { M: 107/204 (52.5) } \\
\text { W: } 97 / 204(47.5)\end{array}$ & $\begin{array}{l}M: 55 / 103(53.4) \\
\text { W: 48/103 (46.6) }\end{array}$ & $\begin{array}{l}M: 52 / 101(51.5) \\
W: 49 / 101(48.5)\end{array}$ & 0.784 \\
\hline \multicolumn{13}{|l|}{$\mathrm{BMl}, \mathrm{kg} / \mathrm{m}^{2}$} \\
\hline \multicolumn{2}{|l|}{ Tobacco use } & $3 / 74(4.23)$ & $38 / 577(6.59)$ & 0.610 & $6 / 95(6.3)$ & $5 / 58(8.6)$ & $1 / 37(2.7)$ & 0.40 & & & & \\
\hline \multicolumn{2}{|l|}{ Alcohol use } & & & & $9 / 95(9.5)$ & $6 / 58(10.3)$ & $3 / 37(8.1)$ & 1.00 & & & & \\
\hline \multicolumn{2}{|l|}{ Any comorbidity } & $25 / 74(33.78)$ & $153 / 577(26.52)$ & 0.212 & & & & & & & & \\
\hline \multicolumn{13}{|l|}{ Coronary artery disease } \\
\hline \multicolumn{13}{|l|}{ Congestive heart failure } \\
\hline \multicolumn{13}{|l|}{ Arrhythmia } \\
\hline \multicolumn{2}{|l|}{ Hypertension } & $12 / 74(16.22)$ & $88 / 577(15.25)$ & 0.864 & $16 / 95(16.8)$ & $10 / 58(17.2)$ & $6 / 37(16.2)$ & 0.90 & & & & \\
\hline \multicolumn{2}{|l|}{ Diabetes mellitus } & $7 / 74(9.46)$ & $41 / 577(7.11)$ & 0.477 & $6 / 95(6.3)$ & $3 / 58(5.2)$ & $3 / 27(8.1)$ & 0.67 & & & & \\
\hline \multicolumn{2}{|l|}{$\begin{array}{l}\text { Cerebrovascular } \\
\text { disease }\end{array}$} & & & & $4 / 95(4.2)$ & $3 / 58(5.2)$ & $1 / 37(2.7)$ & 1.00 & & & & \\
\hline \multicolumn{2}{|l|}{ Pulmonary disease } & $0 / 74(0)$ & $1 / 577(0.17)$ & 1.00 & $5 / 95(5.3)$ & $1 / 58(1.7)$ & $4 / 37(10.8)$ & 0.074 & $9 / 204(4.41)$ & $7 / 103(6.80)$ & 2/101 (1.98) & 0.182 \\
\hline \multicolumn{2}{|l|}{ Chronic renal disease } & $0 / 74(0)$ & $6 / 577(1.04)$ & 1.00 & $1 / 95(1.1)$ & $1 / 58(1.7)$ & $0 / 37(0)$ & 1.00 & & & & \\
\hline \multicolumn{2}{|l|}{ Chronic liver disease } & $8 / 74(10.81)$ & $17 / 577(2.95)$ & 0.004 & & & & & $1 / 204(0.97)$ & & & \\
\hline \multicolumn{2}{|l|}{ Cancer } & $0 / 74(0)$ & $6 / 577(1.04)$ & 1.00 & $5 / 95(5.3)$ & $4 / 58(6.9)$ & $1 / 37(2.7)$ & 0.65 & $13 / 204(6.37)$ & $8 / 103(7.77)$ & $5 / 101(4.95)$ & 0.410 \\
\hline \multicolumn{2}{|l|}{ Cardiovascular disease } & $1 / 74(1.35)$ & $4 / 577(0.69)$ & 0.454 & & & & & $44 / 204(21.57)$ & $23 / 103(22.3)$ & $21 / 101(20.79)$ & 0.789 \\
\hline Immunossuppression & & $0 / 74(0)$ & 1/577 (0.17) & 1.00 & & & & & & & & \\
\hline Viral RNA in feces & & $3 / 9(33.33)$ & $0 / 577(0)$ & & $31 / 65(47.7)$ & $22 / 42(52.4)$ & $9 / 23(39.1)$ & 0.31 & & & & \\
\hline Oxygen supplementatic & & & & & & & & & & & & \\
\hline $\mathrm{ICU}$ & & $5 / 74(6.76)$ & $12 / 577(2.08)$ & 0.034 & & & & & $16 / 204(7.84)$ & $6 / 103(5.94)$ & $10 / 101(9.90)$ & 0.279 \\
\hline Mechanical ventilation & & $5 / 74(6.76)$ & $12 / 577(2.08)$ & 0.034 & & & & & & & & \\
\hline Death & & & & & $0 / 95(0)$ & $0 / 58(0)$ & $0 / 37(0)$ & & $36 / 204(17.65)$ & $19 / 103(18.45)$ & $17 / 101(16.83)$ & 0.762 \\
\hline & & Wan et a & $1 . .^{(21)}$ & & & Redd et a & $1 . .^{(22)}$ & & Nobel e & et al. ${ }^{(23)}$ & Cholankeril e & t al..$^{(24)}$ \\
\hline & Total & $\begin{array}{c}\text { With GI } \\
\text { symptoms }\end{array}$ & $\begin{array}{l}\text { Without GI } \\
\text { symptoms }\end{array}$ & $p$ value & Total & $\begin{array}{c}\text { With GI } \\
\text { symptoms }\end{array}$ & $\begin{array}{l}\text { Without GI } \\
\text { symptoms }\end{array}$ & p value & Tot & & Total & \\
\hline Patients & 230 & 49 & 181 & & 318 & 195 & 123 & & 278 & 78 & 116 & \\
\hline Age & $47.5(36.8-60)$ & $55(40-65)$ & $46(36-57)$ & 0.017 & $63.4 \pm 16.6$ & $62.3 \pm 15.9$ & $65.0 \pm 17.6$ & 0.16 & 18-30: $31 /$ & $1 / 278(11)$ & $50(35-67$ & \\
\hline & & & & & & & & & 31-50: 69/ & $9 / 278(25)$ & & \\
\hline & & & & & & & & & 51-70: 103 & 3/278 (37) & & \\
\hline & & & & & & & & & $>70: 75 / 2$ & /278 (27) & & \\
\hline Sex & $M: 129 / 230(56)$ & M: 27/49 (55) & M: 102/181 (56) & 0.87 & M: 174/318 (54.7) & M: 102/195 (52.3) & M: 72/123 (58.6) & W: 0.28 & M: 145/2 & $278(52)$ & M: 62/116/5 & 53.4) \\
\hline & W: 101/230 (44) & W: 22/49 (45) & W: 79/181 (22) & & $W: 144 / 318(45.3)$ & W: 93/195 (47.7) & W: 51/123 (35.4) & & W: 133/2 & $278(48)$ & W: 54/116 (4 & \\
\hline BMl. kg/m² & & & & & $30.0 \pm 6.5$ & $30.5 \pm 6.7$ & $29.3 \pm 6.2$ & 0.11 & $<25.0: 25$ & $5 / 278(9)$ & $25.8(23.2-3$ & \\
\hline & & & & & & & & & $25.0-29.9: 4$ & $43 / 278(16)$ & & \\
\hline & & & & & & & & & $\geq 30.0: 47$ & 7/278 (17) & & \\
\hline & & & & & & & & & Without evaluatio & on: $165 / 278$ (59) & & \\
\hline Tobacco use & & & & & $35 / 318(11.0)$ & $23 / 195(11.8)$ & $12 / 123(9.8)$ & 0.57 & & & $\begin{array}{l}\text { Former smoker: } \\
\text { Smoker: } 3\end{array}$ & $\begin{array}{l}21(23.3) \\
2.8)\end{array}$ \\
\hline Alcohol use & & & & & $39 / 318(12.3)$ & $28 / 195(14.4)$ & $11 / 123(8.9)$ & 0.15 & & & & \\
\hline Any comorbidity & $58 / 230(25)$ & $19 / 49(39)$ & $39 / 181(22)$ & 0.017 & & & & & & & & \\
\hline Coronary disease & & & & & $46 / 318(14.5)$ & $26 / 195(13.3)$ & $20 / 123(16.3)$ & & & & & \\
\hline $\begin{array}{l}\text { Congestive heart } \\
\text { failure }\end{array}$ & & & & & $31 / 318(9.8)$ & $16 / 195(8.3)$ & $15 / 123(12.2)$ & 0.25 & & & & \\
\hline
\end{tabular}


...Continuation

Table 1. General data of patients with COVID-19 of the nine retrospective studies

\begin{tabular}{|c|c|c|c|c|c|c|c|c|c|c|}
\hline & \multicolumn{4}{|c|}{ Wan et al..$^{(21)}$} & \multicolumn{4}{|c|}{ Redd et al. ${ }^{(22)}$} & \multirow{2}{*}{$\begin{array}{c}\text { Nobel et al. }{ }^{(23)} \\
\text { Total }\end{array}$} & \multirow{2}{*}{$\begin{array}{c}\text { Cholankeril et al. }{ }^{(24)} \\
\text { Total }\end{array}$} \\
\hline & Total & $\begin{array}{l}\text { With GI } \\
\text { symptoms }\end{array}$ & $\begin{array}{l}\text { Without GI } \\
\text { symptoms }\end{array}$ & p value & Total & $\begin{array}{c}\text { With GI } \\
\text { symptoms }\end{array}$ & $\begin{array}{l}\text { Without GI } \\
\text { symptoms }\end{array}$ & $p$ value & & \\
\hline Arrhythmia & & & & & 48/318 (15.1) & 26/195 (13.3) & $22 / 123(17.9)$ & 0.27 & & \\
\hline Hypertension & $40(17)$ & & & & $188 / 318(59.1)$ & $111 / 195(56.9)$ & $77 / 123(62.6)$ & 0.32 & & $32(27.8)$ \\
\hline Diabetes mellitus & $11(5)$ & & & & 105/318 (33.1) & $62 / 195(32.0)$ & $43 / 123(35.0)$ & 0.58 & & $19(16.4)$ \\
\hline $\begin{array}{l}\text { Cerebrovascular } \\
\text { disease }\end{array}$ & & & & & $11 / 318(3.5)$ & $8 / 195(4.1)$ & $3 / 123(2.4)$ & 0.42 & & \\
\hline Pulmonary disease (\%) & $7(3)$ & & & & $67 / 318(21.1)$ & $40 / 195(20.5)$ & $27 / 123(22.0)$ & 0.76 & & $24(20.7)$ \\
\hline Chronic renal disease & & & & & 40/318 (13.8) & 20/195 (11.7) & $20 / 123(17.0)$ & 0.20 & & $4(3.5)$ \\
\hline $\begin{array}{l}\text { Chronic hepatic } \\
\text { disease }\end{array}$ & $6(3)$ & & & & & & & & & $3(2.8)$ \\
\hline Cancer & $3(1)$ & & & & & & & & & \\
\hline Cardiovascular disease & $10(4)$ & & & & & & & & & $15(12.9)$ \\
\hline Immunosuppressant & & & & & & & & & & $4(4.3)$ \\
\hline
\end{tabular}

Viral RNA in feces

\begin{tabular}{|c|c|c|c|c|c|c|c|c|c|c|c|c|}
\hline $\begin{array}{l}\text { Oxygen } \\
\text { supplementation }\end{array}$ & & $44 / 49(90)$ & 143/181 (79) & 0.10 & & & & & & & & \\
\hline ICU & & $15 / 49(31)$ & 20/181 (11) & 0.0015 & $35 / 202(17.5)$ & $20(15$ & $15(21.4)$ & 0.28 & & $44 / 278(16)$ & & \\
\hline Mechanical ventilation & & $6 / 49(12)$ & 3/181 (2) & 0.0036 & $26 / 202(13.0)$ & $14(10$ & $12(16.0)$ & 0.22 & & & & \\
\hline \multirow[t]{3}{*}{ Death } & & $4 / 49(8)$ & $2 / 181(1)$ & 0.020 & $32 / 202(15.8)$ & $16 / 12$ & $16(22.5)$ & 0.06 & & $9 / 278(3.2)$ & & \\
\hline & \multicolumn{2}{|c|}{ Zhang et al. ${ }^{(25)}$} & \multicolumn{10}{|c|}{ Zhou et al. ${ }^{(26)}$} \\
\hline & Total & & Total & $\begin{array}{c}\text { Total medical } \\
\text { team }\end{array}$ & $\begin{array}{r}\text { With GI sympton } \\
\text { medical team }\end{array}$ & ms - & $\begin{array}{l}\text { No GI symptoms - } \\
\text { medical team }\end{array}$ & p value & $\begin{array}{l}\text { Total non- } \\
\text { medical }\end{array}$ & $\begin{array}{c}\text { With GI } \\
\text { symptoms - } \\
\text { non-medical }\end{array}$ & $\begin{array}{l}\text { Without GI } \\
\text { symptoms - } \\
\text { non-medical }\end{array}$ & p value \\
\hline Patients & 140 & & 254 & 93 & 23 & & 70 & & 161 & 43 & 118 & \\
\hline Age & $57(25-87)$ & & $50(36-65)$ & $36(31-41)$ & $35(30-40)$ & & $36(31-42)$ & 0.614 & $62(49-69)$ & $61(49-67)$ & $62(49-70)$ & 0.615 \\
\hline Sex & $\begin{array}{l}\text { M: } 71 / 140 / 50 \\
W: 69 / 140(4 S\end{array}$ & & $\begin{array}{l}\text { M: 115/254 (45.3) } \\
\text { W: 139/254 (54.7) }\end{array}$ & $\begin{array}{l}\text { M: } 32 / 93(34) \\
\text { W: } 61 / 93(66)\end{array}$ & $\begin{array}{l}\text { M: 6/23 (26) } \\
\text { W:17/23 (74) }\end{array}$ & & $\begin{array}{l}M: 26 / 70(37) \\
W: 44 / 70(63)\end{array}$ & 0.45 & $\begin{array}{l}\text { M: 83/161 (52) } \\
\text { W: 78/161 (48) }\end{array}$ & $\begin{array}{l}\text { M: } 16 / 43(37) \\
\text { W: } 27 / 43(63)\end{array}$ & $\begin{array}{l}\text { M: } 67 / 118(57) \\
\text { W: 51/118(43) }\end{array}$ & 0.033 \\
\hline
\end{tabular}

$\mathrm{BMl}, \mathrm{kg} / \mathrm{m}^{2}$

Tobacco use $\quad$ Former smoker: $7 / 140(5.0)$
$\quad$ Smoker: $2 / 140(1.4)$

Alcohol use

\begin{tabular}{|c|c|c|c|c|c|c|c|c|c|c|}
\hline Any comorbidity & $90 / 140(64.3)$ & & & & & & & & & \\
\hline Coronary disease & $7 / 140(5.0)$ & $17 / 254(6.7)$ & $2 / 93(2.1)$ & $0 / 23(0)$ & $2 / 70(3)$ & 1.00 & $15 / 161(9)$ & $6 / 43(14)$ & $9 / 118(8)$ & 0.231 \\
\hline \multicolumn{11}{|l|}{ Congestive heart failure } \\
\hline Arrhythmia & $5 / 140(3.6)$ & & & & & & & & & \\
\hline Hypertension & $42 / 140(30.0)$ & $63 / 254(24.8)$ & $6 / 93(6.4)$ & $0 / 23(0)$ & $6 / 70(9)$ & 0.33 & $57 / 161(35)$ & $14 / 43(33)$ & $43 / 118(36)$ & 0.712 \\
\hline Diabetes mellitus & $17 / 140(12.1)$ & $26 / 254(10.2)$ & $3 / 93(3.2)$ & $0 / 23(0)$ & $3 / 70(4)$ & 0.572 & $23 / 161(14)$ & $4 / 43(9)$ & $19 / 118(16)$ & 0.321 \\
\hline Cerebrovascular disease & $3 / 140(2.1)$ & 13/254 (5.1) & $1 / 93(1.1)$ & $0 / 23(0)$ & $1 / 70(1)$ & 1.00 & 12/161 (7) & $3 / 43(7)$ & $9 / 118(8)$ & 1.00 \\
\hline Pulmonary disease & $2 / 140(1.4)$ & $6 / 254(2.4)$ & $1 / 93(1.1)$ & $0 / 23(0)$ & $1 / 70(1)$ & 1.00 & $5 / 161(3)$ & $2 / 43(5)$ & $3 / 118(3)$ & 0.61 \\
\hline Chronic renal disease & $2 / 140(1.4)$ & & & & & & & & & \\
\hline Chronic liver disease & & $3 / 254(1.2)$ & $1 / 93(1.1)$ & $0 / 23(0)$ & $1 / 70(1)$ & 1.00 & $2 / 161(1)$ & $0 / 43(0)$ & $2 / 118(2)$ & 1.00 \\
\hline Cancer & & $2 / 254(0.8)$ & $1 / 93(1.1)$ & $0 / 23(0)$ & $1 / 70(1)$ & 1.00 & $1 / 161(1)$ & $0 / 43(0)$ & $1 / 118(1)$ & 1.00 \\
\hline \multicolumn{11}{|l|}{ Cardiovascular disease } \\
\hline Immunosuppression & & $1 / 254(0.4)$ & $0 / 93(0)$ & $0 / 23(0)$ & $0 / 70(0)$ & & $1 / 161(1)$ & $1 / 43(2)$ & $0 / 118(0)$ & 0.267 \\
\hline \multicolumn{11}{|l|}{ Viral RNA in feces } \\
\hline \multicolumn{11}{|l|}{ Oxygen supplementation } \\
\hline \multicolumn{11}{|l|}{ ICU } \\
\hline Mechanical ventilation & & $18 / 254(7)$ & $5 / 93(5.4)$ & $1 / 23(4.3)$ & $4 / 70(5.7)$ & 1.00 & 13/161 (8) & $2 / 43(4.7)$ & $11 / 118(9.3)$ & 0.516 \\
\hline Death & & $16 / 254(6.3)$ & $2 / 93(2.1)$ & $1 / 23(4.3)$ & $1 / 70(1.43)$ & 0.435 & $14 / 161(9)$ & $3 / 43(7)$ & $11 / 118(9.3)$ & 0.457 \\
\hline
\end{tabular}

Results expressed as $n$. mean \pm standard deviation. $n / n$ total $(\%)$.

GI: gastrointestinal; M: men; W: women; BMI: body mass index; ICU: intensive care unit. 
Table 2. Typical symptoms of patients with COVID-19 in the nine retrospective studies

\begin{tabular}{|c|c|c|c|c|c|c|c|c|c|c|c|c|}
\hline \multirow[b]{2}{*}{ Symptoms } & \multicolumn{3}{|c|}{ Jin et al. ${ }^{(18)}$} & Lin et al. ${ }^{(19)}$ & \multicolumn{3}{|c|}{ Pan et al. ${ }^{(20)}$} & \multirow{2}{*}{$\frac{\text { Wan et al. }{ }^{(21)}}{\text { Total }}$} & \multicolumn{4}{|c|}{ Redd et al. ${ }^{(22)}$} \\
\hline & $\begin{array}{c}\text { With GI } \\
\text { symptoms }\end{array}$ & $\begin{array}{l}\text { Without GI } \\
\text { symptoms }\end{array}$ & p value & Total & Total & $\begin{array}{l}\text { With GI } \\
\text { ymptoms }\end{array}$ & $\begin{array}{l}\text { Without GI } \\
\text { symptoms }\end{array}$ & & Total & $\begin{array}{c}\text { With GI } \\
\text { symptoms }\end{array}$ & $\begin{array}{l}\text { Without GI } \\
\text { symptoms }\end{array}$ & $\mathrm{p}$ value \\
\hline Patients & 74 & 577 & & 95 & 204 & 103 & 101 & 230 & 318 & 195 & 123 & \\
\hline Fever & $\begin{array}{c}63 / 74(85.14) \\
>38.5^{\circ} \mathrm{C}: \\
29 / 74(39.19)\end{array}$ & $\begin{array}{c}482 / 577(83.54) \\
>38.5^{\circ} \mathrm{C}: \\
101 / 577(17.50)\end{array}$ & $\begin{array}{c}0.867 \\
>38.5^{\circ} \mathrm{C} \\
<0.001\end{array}$ & Yes & & $5 / 103(92.23)$ & & 193/230 (84) & $258 / 318(81.3)$ & $161 / 195(82.6)$ & $97 / 123(78.9)$ & 0.41 \\
\hline Fatigue & $23 / 74(31.08)$ & $96 / 577(16.64)$ & 0.004 & & & $4 / 103(52.42)$ & & $43(19)$ & $183 / 318(57.5)$ & $127 / 195(65.1)$ & $56 / 123(45.5)$ & 0.0006 \\
\hline Myalgia & 10/74 (13.51) & $61 / 577(10.57)$ & 0.430 & & & $5 / 103(14.56)$ & & & $123 / 318(38.7)$ & $96 / 195(49.2)$ & $27 / 123(22.0)$ & $<0.0001$ \\
\hline Chills & & & & & & & & & $72 / 318(22.6)$ & $50 / 195(25.6)$ & $22 / 123(17.9)$ & 0.11 \\
\hline Diaphoresis & & & & & & & & & $15 / 318(4.7)$ & $12 / 195(6.2)$ & $3 / 123(2.4)$ & 0.13 \\
\hline Arthralgia & & & & & & & & & $8 / 318(2.5)$ & $4 / 195(2.1)$ & $4 / 123(3.3)$ & 0.51 \\
\hline Dry cough & $53 / 74(71.62)$ & $382 / 577(66.20)$ & 0.431 & & & & & $159 / 230(69)$ & $247 / 318(77.7)$ & $156 / 195(80.0)$ & $91 / 123(74.0)$ & 0.21 \\
\hline $\begin{array}{l}\text { Productive } \\
\text { cough }\end{array}$ & $29 / 74(39.19)$ & 198/577 (34.32) & 0.438 & & & & & $98 / 230(43)$ & $45 / 318(14.2)$ & $33 / 195(16.9)$ & $12 / 123(9.8)$ & 0.74 \\
\hline Dyspnea & $8 / 74(10.81)$ & 19/577 (3.30) & 0.007 & & & & & $30(13)$ & $191 / 318(60.1)$ & $107 / 195(54.9)$ & $84 / 123(68.3)$ & 0.02 \\
\hline Sore throat & $6 / 74(8.11)$ & $93 / 577(16.12)$ & 0.085 & & & & & & $54 / 318(17.0)$ & $42 / 195(21.5)$ & $12 / 123(9.8)$ & 0.0064 \\
\hline Rhinorrhea & & & & & & & & & $36 / 318$ (11.4) & $26 / 195(13.4)$ & 10/123(8.1) & 0.15 \\
\hline $\begin{array}{l}\text { Nasal } \\
\text { obstruction }\end{array}$ & $2 / 74(2.70)$ & $35 / 577(6.07)$ & 0.419 & & & & & & & & & \\
\hline \multicolumn{13}{|l|}{ Dizziness } \\
\hline Anosmia & & & & & & & & & $32 / 318(10.1)$ & $26 / 195(13.3)$ & $6 / 123(4.9)$ & 0.0146 \\
\hline Ageusia & & & & & & & & & $24 / 318(7.6)$ & $21 / 195(10.9)$ & $3 / 123(2.4)$ & 0.0057 \\
\hline Headache & $16 / 74(21.62)$ & $51 / 577(8.84)$ & 0.002 & & & & & $19(8)$ & & & & \\
\hline Hemoptysis & $3 / 74(4.05)$ & $8 / 577(1.39)$ & 0.119 & & & & & $3(1)$ & & & & \\
\hline \multirow[b]{2}{*}{ Symptoms } & Nobel et al. ${ }^{(23)}$ & Cholankeril et al. ${ }^{(24)}$ & Zhang et al. ${ }^{(25)}$ & \multicolumn{9}{|c|}{ Zhou et al. ${ }^{(26)}$} \\
\hline & Total & Total & Total & Total & $\begin{array}{c}\text { Total - } \\
\text { Medical } \\
\text { team }\end{array}$ & $\begin{array}{c}\text { With GI } \\
\text { symptoms - } \\
\text { medical team }\end{array}$ & $\begin{array}{c}\text { Without GI } \\
\text { symptoms - } \\
\text { medical team }\end{array}$ & p value & $\begin{array}{l}\text { Total non- } \\
\text { medical }\end{array}$ & $\begin{array}{c}\text { With GI } \\
\text { symptoms - } \\
\text { non-medical }\end{array}$ & $\begin{array}{l}\text { Without GI } \\
\text { symptoms - } \\
\text { non-medical }\end{array}$ & p value \\
\hline Patients & 278 & 116 & 140 & 254 & 93 & 23 & 70 & & 161 & 43 & 118 & \\
\hline Fever & & 76.70 & $110 / 120(91.7)$ & $213 / 254(83.9)$ & 9) $80 / 93(86)$ & 19/23 (83) & $61 / 70(87)$ & 0.729 & 133/161 (82) & $39 / 43(91)$ & $94 / 118(80)$ & 0.157 \\
\hline Fatigue & & & $90 / 120(75.0)$ & $133 / 254(52.4)$ & 4) $52 / 93(56)$. & $12 / 23(52)$ & $40 / 70(57)$ & 0.809 & $81 / 161(50)$ & $29 / 43(67)$ & $52 / 118(44)$ & 0.012 \\
\hline Myalgia & & 52.50 & & $86 / 254(33.9)$ & ) $\quad 41 / 93(44)$. & $10 / 23(44)$ & $31 / 70(44)$ & 1 & $45 / 161(28)$ & $17 / 43(40)$ & $28 / 118(24)$ & 0.073 \\
\hline \multicolumn{13}{|l|}{ Chills } \\
\hline \multicolumn{13}{|l|}{ Diaphoresis } \\
\hline \multicolumn{13}{|l|}{ Arthralgia } \\
\hline Dry cough & & 94.8 & $90 / 120(75.0)$ & $98 / 254(38.6)$ & ) $\quad 41 / 93(44)$ & $7 / 23(30)$ & $34 / 70(49)$ & 0.152 & $57 / 161(35)$ & $14 / 43(33)$ & $43 / 118(36)$ & 0.712 \\
\hline $\begin{array}{l}\text { Productive } \\
\text { cough }\end{array}$ & & & & $107 / 254(42.1)$ & 1) $31 / 93(33)$ & $6 / 23(26)$ & $25 / 70(36)$ & 0.454 & $76 / 161(47)$ & $17 / 43(40)$ & $59 / 118(50)$ & 0.286 \\
\hline Dyspnea & & 58 & $44 / 120(36.7)$ & 10/254 (3.9) & $2 / 93(2.1)$ & $1 / 23(4)$ & $1 / 70(1)$ & 0.435 & $8 / 161(5.0)$ & $2 / 43(5)$ & $6 / 118(5)$ & 1.00 \\
\hline Sore throat & & & & $16 / 254(6.3)$ & $6 / 93(6.4)$ & $0 / 23(0)$ & $6 / 70(9)$ & 0.33 & 10/161 (6.2) & $6 / 43(14)$ & $4 / 118(3)$ & 0.023 \\
\hline \multicolumn{13}{|l|}{ Rhinorrhea } \\
\hline \multicolumn{13}{|c|}{ Nasal obstruction } \\
\hline Dizziness & & & & $18 / 254(7.1)$ & 10/93 (11) & $4 / 23(17)$ & $6 / 70(9)$ & 0.256 & $8 / 161(5.0)$ & $5 / 43(12)$ & $3 / 118(3)$ & 0.032 \\
\hline \multicolumn{13}{|l|}{ Anosmia } \\
\hline \multicolumn{13}{|l|}{ Ageusia } \\
\hline \multicolumn{13}{|l|}{ Headache } \\
\hline Hemoptysis & & & & & & & & & & & & \\
\hline
\end{tabular}

Results expressed as $n$, mean \pm standard deviation, $n / n$ total $(\%)$.

GI: gastrointestinal. 
Table 3. Gastrointestinal symptoms of patients with COVID-19 in the nine retrospective studies

\begin{tabular}{|c|c|c|c|c|c|c|c|c|c|c|c|c|c|}
\hline & \multirow[b]{2}{*}{ Jin et al..$^{(18)}$} & \multicolumn{3}{|c|}{ Lin et al. ${ }^{(19)}$} & \multirow[b]{2}{*}{$\begin{array}{l}\text { Pan L et } \\
\text { al. }{ }^{(20)}\end{array}$} & \multirow[b]{2}{*}{$\begin{array}{c}\text { Wan } \\
\text { et al. }{ }^{(21)}\end{array}$} & \multirow[b]{2}{*}{$\begin{array}{l}\text { Redd } \\
\text { et al. }{ }^{(22)}\end{array}$} & \multirow[b]{2}{*}{$\begin{array}{l}\text { Nobel et } \\
\text { al. }{ }^{(23)}\end{array}$} & \multirow[b]{2}{*}{$\begin{array}{c}\text { Cholankeril } \\
\text { et al. }{ }^{(24)}\end{array}$} & \multirow[b]{2}{*}{$\begin{array}{l}\text { Zhang } \\
\text { et al. }{ }^{25)}\end{array}$} & \multicolumn{3}{|c|}{ Zhou et al. ${ }^{(26)}$} \\
\hline & & Total & $\begin{array}{c}\text { At } \\
\text { admission }\end{array}$ & $\begin{array}{c}\text { During } \\
\text { hospitalization }\end{array}$ & & & & & & & Total & $\begin{array}{c}\text { With GI } \\
\text { symptoms - } \\
\text { medical team }\end{array}$ & $\begin{array}{c}\text { With GI } \\
\text { symptoms - } \\
\text { GI non-medical }\end{array}$ \\
\hline Patients & 651 & & 95 & & 204 & 230 & 318 & 278 & 116 & 140 & 254 & 23 & 43 \\
\hline GIT symptoms & $74 / 651$ (11.4) & $58 / 95(61.1)$ & $11 / 95(11.6)$ & $47 / 95(49.5)$ & $103 / 204(50.5)$ & $\begin{array}{c}49 / 230 \\
(21)\end{array}$ & $\begin{array}{c}195 / 318 \\
(61.3)\end{array}$ & 97/278 (35) & $37(31.9)$ & $\begin{array}{l}55 / 139 \\
(39.6)\end{array}$ & $66 / 254(26.0)$ & $23 / 23(100)$ & $43 / 43(100)$ \\
\hline Diarnea & $53 / 651$ (8.14) & $23 / 95(24.2)$ & $5 / 95(5.3)$ & $18 / 95(18.9)$ & $35 / 103(33.98)$ & $\begin{array}{c}49 / 230 \\
(21)\end{array}$ & $\begin{array}{c}107 / 318 \\
(33.7)\end{array}$ & $56 / 278(20)$ & $12(10.3)$ & $\begin{array}{l}18 / 139 \\
(12.9)\end{array}$ & $46 / 254(18.1)$ & $19 / 23(86.6)$ & $27 / 43(62.8)$ \\
\hline \multirow[t]{2}{*}{$\mathrm{N} N$} & $\begin{array}{c}\mathrm{N}: 10 / 651 \\
(1.53)\end{array}$ & $\mathrm{N}: 17 / 95(17.9)$ & $\mathrm{N}: 3 / 95(3.2)$ & $\mathrm{N}: 14 / 95(14.7)$ & V: 4/103 (3.88) & & $\begin{array}{c}\mathrm{N}: 84 / 318 \\
(26.4)\end{array}$ & $63 / 278(23)$ & $12(10.3)$ & $\begin{array}{c}N: 24 / 139 \\
(17.3)\end{array}$ & $N: 21 / 254(8.3)$ & N: 5/23 (21.7) & $N: 16 / 43(37.2)$ \\
\hline & $\begin{array}{c}V: 11 / 651 \\
(1.69)\end{array}$ & $V: 4 / 95(4.2)$ & $V: 0 / 95(0)$ & $V: 4 / 95(4.2)$ & & & $\begin{array}{c}V: 49 / 318 \\
(15.4)\end{array}$ & & & $\begin{array}{c}V: 7 / 139 \\
(5.0)\end{array}$ & V: $15 / 254(5.9)$ & $V: 1 / 23(4.3)$ & $V: 14 / 43(32.6)$ \\
\hline Anorexia & & $17 / 95(17.9)$ & $5 / 95(5.3)$ & $12 / 95(12.6)$ & $81 / 103(78.64)$ & & $\begin{array}{c}110 / 318 \\
(34.8)\end{array}$ & & $22(25.3)$ & $\begin{array}{l}17 / 139 \\
(12.1)\end{array}$ & & & \\
\hline Abdominal pain & & $2 / 95(2.1)$ & $0 / 95(0)$ & $2 / 95(2.1)$ & $2 / 103(1.94)$ & $3 / 230(1)$ & $46 / 318(14.5)$ & & $10(8.8)$ & $\begin{array}{r}8 / 139 \\
(5.8)\end{array}$ & $3 / 254(1.2)$ & $0 / 23(0)$ & $3 / 43(7.0)$ \\
\hline Weight loss & & & & & & & $30 / 318(9.4)$ & & & & & & \\
\hline Melena & & & & & & $10 / 230(4)$ & $2 / 318(0.63)$ & & & & & & \\
\hline Reflux & & $2 / 95(2.1)$ & $1 / 95(1.1)$ & $1 / 95(1.1)$ & & & $2 / 318(0.63)$ & & & & & & \\
\hline Dysphagia & & & & & & & $1 / 318(0.31)$ & & & & & & \\
\hline Odynophagia & & & & & & & $1 / 318(0.31)$ & & & & & & \\
\hline Hematochezia/UGIB & & $2 / 95(2.1)$ & $0 / 95(0)$ & $2 / 95(2.1)$ & & & $1 / 318(0.31)$ & & & & & & \\
\hline Constipation & & & & & & & $3 / 318(0.94)$ & & & & & & \\
\hline
\end{tabular}

Results expressed as $n$ or $n / n$ total $(\%)$.

GI: gastrointestinal; GIT: gastrointestinal tract; N: nausea; V: vomiting; UGIB: upper gastrointestinal bleeding.

Jin et al., did a retrospective study in China that analyzed 651 patients (331 men and 320 women), mean age of $46.14 \pm 14.19$ years, with confirmed with COVID-19, in the Zhejiang province. Of these, 74 $(11.4 \%)$ presented with at least one gastrointestinal symptom, such as nausea, vomiting, and diarrhea at hospital admission. The most common gastrointestinal symptom was diarrhea $(8.14 \%)$, with a mean duration of 4 days, and most of the cases were self-limited. Of the patients with gastrointestinal symptoms, 29 had significantly higher rates of fever $>38.5^{\circ} \mathrm{C}(39.19 \%$ versus $17.50 \%$ of those without gastrointestinal symptoms; $\mathrm{p}<0.001), 23$ fatigue ( $31.08 \%$ versus $16.64 \% ; \mathrm{p}=0.004)$, eight dyspnea $(10.81 \%$ versus $3.30 \% ; \mathrm{p}=0.007)$, and 16 headache $(21.62 \%$ versus $8.84 \% ; \mathrm{p}=0.002)$. There was also a significant difference between patients with chronic liver disease $(10.81 \%$ with gastrointestinal symptoms versus $2.95 \%$ without gastrointestinal symptoms; $p=0.004$ ) and severity of the disease at admission, with respiratory failure, and shock. In addition, multiple organ failure requiring mechanical ventilation and admission to the intensive care unit (ICU; $22.97 \%$ versus $8.14 \%$; $\mathrm{p}<0.001$ ). Regarding test findings, serum sodium levels were lower in patients with gastrointestinal symptoms $(137.65 \mathrm{mmol} / \mathrm{L}$ versus $138.33 \mathrm{mmol} / \mathrm{L} ; \mathrm{p}=0.016)$, with a tendency towards having more severe disease. Aspartate aminotransferase (AST) had a higher rate in these patients $(29.35 \mathrm{U} / \mathrm{L}$ versus $24.4 \mathrm{U} / \mathrm{L} ; \mathrm{p}=0.02$ ). Patients with gastrointestinal symptoms had more significant complications during treatment, such as progression to SARS with need of ICU admission (6.67\% versus $2.08 \% ; \mathrm{p}=0.034)$, and liver injury $(17.57 \%$ versus $8.84 \%$; $p=0.035)$, than did those without gastrointestinal symptoms. The presence of viral RNA in feces was detected in few patients - only three of nine patients with gastrointestinal symptoms. Because of the small sample, they did not evaluate the implications of oral-fecal transmission, which needs further investigation. It is worth remembering, however, that this study had some limitations. It was not a cohort and did not identify patients with gastrointestinal symptoms who did not have the typical symptoms of cough and fever. ${ }^{(18)}$

In the study of Lin et al., 95 patients (50 women and 45 men) from Zhuhai, China, with COVID-19 and mean age of $45.3 \pm 18.3$ years, $58(61.0 \%)$ presented with gastrointestinal symptoms, in which 11 (11.6\%) at hospital admission and 47 (49.5\%) during hospitalization. The latter probably had the condition aggravated by use of drugs, such as antibiotics. The main gastrointestinal symptoms were diarrhea, anorexia, and nausea, in $24.2 \%$, $17.9 \%$, and $17.9 \%$, respectively. The presence of RNA 
Table 4. Typical and gastrointestinal symptoms of patients with COVID-19 in the meta-analyses

\begin{tabular}{|c|c|c|c|c|c|}
\hline & Sultan et al..$^{(8)}$ & Cheung et al. ${ }^{(27)}$ meta-analysis & Cheung et al. ${ }^{(27)}$ Hong Kong cohort & Cao et al..$^{(28)}$ & Li et al. ${ }^{(29)}$ \\
\hline Patients & 10,890 & 4,243 & 59 & 46,959 & 1,994 \\
\hline Age & & 45.1 (IOR: $41.0-54.8)$ & 58.5 (IQR: 43.5-68.0; range: 22-96) & 46.62 (95\% Cl: $31.710-61.531)$ & \\
\hline Sex & & M: 57.3 & M: $27 / 59(45.8)$ & M: 55.6 (95\%Cl: 0.530-0.602) & M: 60 (95\%Cl: 0.54-0.65) \\
\hline Comorbidities & & & & $35.6(95 \%$ Cl: $0.267-0.444)$ & \\
\hline \multirow[t]{6}{*}{ Gl symptoms } & & Total: 17.6 (95\%Cl: 12.3-24.5) & $15 / 59(25,4)$ & $6.8(95 \% \mathrm{Cl}: 0.044-0.092)$ & \\
\hline & & 60 studies and 4,243 patients & & & \\
\hline & & China: 16.1 (95\%Cl: 10.9-23.0) & & & \\
\hline & & 53 studies and 4,198 patients & & & \\
\hline & & Other countries: 33.4 (95\% Cl: 15.2-58.3) & & & \\
\hline & & 7 studies and 45 patients & & & \\
\hline \multirow[t]{2}{*}{ Anorexia } & & $26.8(95 \% \mathrm{Cl}: 16.2-40.8)$ & & & \\
\hline & & 18 studies & & & \\
\hline \multirow[t]{6}{*}{ Nausea/vomiting } & Total: 7.8 (7.1-8.5) & $10.2(95 \% \mathrm{Cl}: 6.6-15.3)$ & $1 / 59(1,7)$ & & 3.90 \\
\hline & 26 studies and 5,955 patients & 32 studies & & & \\
\hline & China: $5.2(4.4-5.9)$ & & & & \\
\hline & 19 studies and 4,054 patients & & & & \\
\hline & Other countries: $14.9(13.3-16.6)$ & & & & \\
\hline & 7 studies and 1,901 patients & & & & \\
\hline \multirow[t]{6}{*}{ Diarrhea } & Total: $7.7(7.2-8.2)$ & $12.5(95 \%$ Cl: $9.6-16.0)$ & $13 / 59(22.0)$ & $6.8(95 \% \mathrm{Cl}: 0.044-0.092)$ & 4.80 \\
\hline & 43 studies and 10,676 patients & & & & \\
\hline & China: $5.8(5.3-6.4)$ & & & & \\
\hline & 32 studies and 8,612 patients & & & & \\
\hline & Other countries: $18.3(16.6-20.1)$ & & & & \\
\hline & 11 studies and 2,064 patients & & & & \\
\hline \multirow[t]{6}{*}{ Abdominal pain } & Total: $3.6(3.0-4.3)$ & $9.2(95 \% \mathrm{Cl}: 5.7-14.5)$ & $7 / 59(11.9)$ & & \\
\hline & 15 studies and 4,031 patients & 12 studies & & & \\
\hline & China: $2.7(2.0-3.4)$ & & & & \\
\hline & 10 studies and 2,447 patients & & & & \\
\hline & Other countries: $5.3(4.2-6.6)$ & & & & \\
\hline & 5 studies and 1,584 patients & & & & \\
\hline \multirow[t]{3}{*}{ PCR in feces } & $15 / 50(30)$ & $48.1 \%$ (95\%Cl: $38.33-57.94)$ & $9 / 59(15.3)$ & & \\
\hline & $7 / 10(70)$ & 12 studies and 138 patients & & & \\
\hline & $44 / 153(29)$ & & & & \\
\hline Fever & & & $56 / 59(94.9)$ & 87.3 (95\%Cl: 0.838-0.909) & 88.50 \\
\hline Dry cough & & & $22 / 59(37.3)$ & 58.1 (95\%Cl: 0.502-0.060) & 68.60 \\
\hline Sore throat & & & & 12.0 (95\%Cl: $0.062-0.177)$ & \\
\hline Productive cough & & & & $29.4(95 \%$ Cl: $0.171-0.417)$ & 28.20 \\
\hline Chest pain & & & & 31.2 (95\%Cl: $-0.024-0.648)$ & \\
\hline Myalgia or fatigue & & & & 35.5 (95\%Cl: $0.253-0.456)$ & 35.80 \\
\hline $\begin{array}{l}\text { Headache or } \\
\text { dizziness }\end{array}$ & & & & $9.4(95 \% \mathrm{Cl}: 0.063-0.126)$ & 12.20 \\
\hline Dyspnea & & & $4 / 59(6.8)$ & $38.2(95 \% \mathrm{Cl}: 0.246-0.520)$ & 21.90 \\
\hline
\end{tabular}

IQR: interquartile range; Cl: confidence interval; M: men; Gl: gastrointestinal.

of the virus in feces was tested in 65 patients ( 42 with and 23 without gastrointestinal symptoms) and was positive in $22(52.4 \%)$ of those with gastrointestinal symptoms, and $9(39.1 \%)$ of those without. The presence of RNA of the virus in feces does not necessarily indicate more severe gastrointestinal symptoms, because it did not show a significant difference between the two groups $(\mathrm{p}=0.31)$. However, the presence of the virus in the tissue indicated a more severe disease. In all, six patients were submitted to endoscopy (two were severe and four non-severe), and one presented with bleeding in the esophagus with ulcers and erosions. The two severe patients were also submitted to proctoscopy, and the RNA of the virus was detected in the esophagus, 
stomach, duodenum, and rectum of these patients. Of the non-severe patients, only one had viral RNA presente in the duodenum. This study concluded that GIT can be a potential transmission route and target organ for SARS-CoV-2, and gastrointestinal symptoms cannot be underestimated. ${ }^{(19)}$

According to the descriptive, cross-sectional, multicenter study (three hospitals in Hubei, China) by Pan et al., with 204 patients, in which 107 were male, mean age of 52.91 15.98 years, $103(50.5 \%)$ reported some gastrointestinal symptom, such as lack of appetite $(81 ; 78.6 \%)$, diarrhea $(35 ; 34.0 \%)$, vomiting $(4 ; 3.9 \%)$, and abdominal pain $(2 ; 1.9 \%)$. If we eliminate the lack of appetite, there were $38(18.6 \%)$ patients with gastrointestinal symptoms. Six patients (3\%) reported only digestive symptoms. It was noted that with increased severity of the disease, the gastrointestinal symptoms became more pronounced. Patients with gastrointestinal symptoms had more laboratory abnormalities, such as elevation of alanine aminotransferase (ALT; 42.24U/L versus $29.53 \mathrm{U} / \mathrm{L} ; \mathrm{p}=0.011)$ and $\mathrm{AST}(35.12 \mathrm{U} / \mathrm{L}$ versus $27.48 \mathrm{U} / \mathrm{L} ; \mathrm{p}=0.032$ ), decrease in monocyte count $\left(0.39 \times 10^{9} / \mathrm{L}\right.$ versus $\left.\left.0.46 \times 10^{9} / \mathrm{L}\right) ; \mathrm{p}=0.021\right)$, increased prothrombin time (13.13 seconds versus 12.53 seconds; $\mathrm{p}=0.024)$, and received more antimicrobials during treatment $(76.70 \%$ versus $61.39 \% ; \mathrm{p}=0.018)$, when compared to those without gastrointestinal symptoms. Thus, these patients had a greater chance of suffering hepatic injury. Yet, since this was a retrospective study, some limitations should be considered, such as small sample and they did not test the presence of SARS-CoV-2 in the patients' feces. ${ }^{(20)}$

Wan et al., conducted a multicenter retrospective study with 14 hospitals in China and showed gastrointestinal symptoms are common in patients with COVID-19. Of 230 patients with the disease, 129 were men, mean age of 47.5 years, and diarrhea was observed in $49(21 \%)$ of them. Other gastrointestinal symptoms, such as abdominal pain (3.1\%) and melena (10.4\%), were also reported. The patients with diarrhea had more comorbidities ( $39 \%$ versus $22 \%$; $\mathrm{p}=0.017$ ), more advanced age (55 years versus 46 years; $\mathrm{p}=0.017$ ), and presented with more severe symptoms of respiratory problems, requiring ICU ( $31 \%$ versus $11 \%$; $p=0.0015)$, mechanical ventilation $(12 \%$ versus $2 \% ; \mathrm{p}=0.0036)$, and progressing to death ( $8 \%$ versus $1 \% ; \mathrm{p}=0.020)$. The study has no data on asymptomatic patients, which may overestimate the prevalence of gastrointestinal symptoms in COVID-19.(21)

A cohort study by Redd et al., in nine hospitals in Massachusetts, United States, evaluated the presence of gastrointestinal symptoms in 318 adult patients (174 men) with COVID-19 at hospital admission and mean age 63.4 16.6 years. A total of 195 (61.3\%) patients presented with gastrointestinal symptoms upon admission, among which were anorexia (110; $34.8 \%)$, diarrhea $(107 ; 33.7 \%)$, nausea $(84 ; 26.4 \%)$, and vomiting $(49 ; 15.4 \%)$. The general symptoms significantly associated with the gastrointestinal tract were fatigue ( $65.1 \%$ versus $45.5 \%$; $p=0.0006)$, myalgia (49.2\% versus $22.0 \%$; $\mathrm{p}<0.0001)$, sore throat $(21.5 \%$ versus $9.8 \% ; \mathrm{p}=0.0064)$, anosmia $(13.3 \%$ versus $4.9 \%$; $\mathrm{p}=0.0146)$, and ageusia $(10.9 \%$ versus $2.4 \%$; $\mathrm{p}=0.0057)$. A subgroup of 202 patients required hospitalization at the time this article was being written; 35 (17.5\%) went to the ICU, $26(13.0 \%)$ needed mechanical ventilation, and $32(15.8 \%)$ died, but no significant difference was observed with worsening of the clinical picture in patients with and without gastrointestinal symptoms. The limitations of the study were a retrospective design, lack of instruments to validate symptoms, focus only on hospital data, and the fact that outpatients with less severe disease were excluded. In addition, the article did not evaluate the presence of the virus in the stool of these patients. ${ }^{(22)}$

Nobel et al., performed a retrospective control case study that compared the symptoms of 278 patients positive for SARS-CoV-2 with 238 negative patients in the United States. The gastrointestinal symptoms considered were diarrhea or nausea/vomiting. They found a significant difference between gastrointestinal symptoms in patients with and without COVID-19 $(p=0.04)$. The presence of these symptoms was associated to a risk greater than $70 \%$ of having COVID-19. Among the COVID-19 patients, 97 (35\%) presented with gastrointestinal symptoms, such as diarrhea $(56 ; 20 \%)$ and nausea/vomiting $(63 ; 23 \%)$. Patients with gastrointestinal symptoms had a longer duration of the disease when compared to those without these symptoms ( $33 \%$ versus $22 \%$; $\mathrm{p}=0.048$ ). The limitations of the study were a short patient followup period, a retrospective study, and the identification of gastrointestinal symptoms was dependent on documentation done well. This article also did not evaluate the presence of the virus in the feces of the patients. ${ }^{(23)}$

In a cohort study by Cholankeril et al., in an organization in the United States, gastrointestinal symptoms were reported in $31.9 \%$ (97) of patients with COVID-19, and the most common symptoms were loss of appetite $(22 ; 25.3 \%)$, nausea/vomiting (12; $10.3 \%)$, and diarrhea $(12 ; 10.3 \%)$. The study included 
116 patients with COVID-19, most of them male $(53.4 \%)$, mean age of 50 years. No patient presented with isolated gastrointestinal symptoms as the initial manifestation. The mean duration of gastrointestinal symptoms was one day, which was significantly shorter than the duration of respiratory symptoms $(\mathrm{p}<0.001)$. The elevation of AST correlated with severity of the disease $(\mathrm{p}=0.009)$. This study presented limitations, such as patients from only one organization (regional trend), the fact of documentation of extrapulmonary symptoms may be incomplete, and the diagnosis of COVID-19 was only done in patients with respiratory symptoms. The article did not evaluate the presence of the virus in the feces of these patients. ${ }^{(24)}$

For Zhang et al., who studied in 140 patients from China with COVID-19, 39.6\% of them presented with gastrointestinal symptoms. In this study, the proportion between men and women was practically the same, and mean age was 57.0 years. The main symptoms were fever (91.7\%), cough (75.0\%), and fatigue $(75.0 \%)$. They point out that eosinopenia, with or without lymphopenia in patients with symptoms and radiological changes, may be a potential indicator for the diagnosis of COVID-19. In addition, they found that allergic diseases, asthma, and chronic obstructive pulmonary disease (COPD) are not risk factors for SARS-CoV-2 infection, but studies with larger samples are needed to confirm these findings. Advanced age ( $\mathrm{p}<0.001)$, comorbidities $(\mathrm{p}=0.002)$, and laboratory abnormalities are associated with severity of the disease. These laboratory values are high D-dimer $(\mathrm{p}<0.001), \mathrm{C}$-reactive protein $(\mathrm{p}<0.001)$, procalcitonin $(\mathrm{p}<0.001)$, and leukopenia $(\mathrm{p}<0.014)$. In this study, patients with COPD and smokers had a lower risk of being infected by SARS-CoV-2, but the progression of the condition in these patients was more severe. However, the relation of smokers with COVID-19 is still uncertain. Smokers and COPD patients are more susceptible to MERS-CoV infections, because tobacco and COPD increase the expression of the DPP4 carrier, which is the receptor for this virus. ${ }^{(25)}$

Zhou et al., evaluated 254 patients of a Wuhan center with confirmation of COVID-19. They compared the infected medical team separately from the non-medical patients. In the sample, 139 were women, mean age was 50.6 years, and 161 did not belong to the medical team. The most frequently reported symptoms in all patients were fever $(211 ; 83 \%)$, cough $(98 ; 38.6 \%)$, and gastrointestinal symptoms $(66 ; 26 \%)$. In non-medical patients, the proportion of gastrointestinal symptoms in women was significantly higher than in men (62.8\% versus $37.2 \%$; $p=0.033)$. In addition, clinical manifestations, such as sore throat (14\% versus 3\%; $\mathrm{p}=0.023)$, dizziness $(12 \%$ versus $3 \% ; \mathrm{p}=0.032)$, fatigue (67\% versus $44 \% ; p=0.012)$, low hemoglobin (116.7 versus $133 ; \mathrm{p}=0.028)$, high $\mathrm{C}$-reactive protein $(7.3$ versus $3.8 ; \mathrm{p}=0.021)$, and high ALT (64.1 versus 46.6; $\mathrm{p}=0.049$ ), were also significantly more frequent in non-medical patients with gastrointestinal symptoms. Nonetheless, there was no significant correlation between symptoms and clinical characteristics of patients with and without gastrointestinal symptoms. An explanation of why the medical team was less susceptible to gastrointestinal symptoms is that most of this group was made up of young nurses with no comorbidities. This study has limitations. Most of the cases included were clinically confirmed, therefore it has patients without the PCR test for SARS-CoV-2. Additionally, many patients did not have follow-up, since they were still hospitalized at the time of submission of this article. ${ }^{(26)}$

Cipriano et al., conducted a systematic review with six studies of patients from China, which points to the possibility of SARS-CoV-2 infection in the gastrointestinal tract and fecal-oral transmission. In the study, $53.42 \%$ of stool samples tested positive for the presence of SARS-CoV-2 RNA in 73 hospitalized patients. These samples remained positive between one and 12 days, and $23.29 \%$ of patients had feces positive for the virus RNA after the respiratory samples tested negative. The authors conclude that the anal swab can be as important as the nasopharyngeal swab, even in asymptomatic patients. Before hospital discharge, physicians should consider that gastrointestinal infection and potential fecal-oral transmission may remain until after the viral disappearance of the respiratory tract. ${ }^{(30)}$

Cheung et al., did an interesting study on gastrointestinal symptoms of COVID-19 and the presence of viral RNA in feces. They compared a metaanalysis with a Hong Kong cohort. In a cohort of 59 patients with the disease, $15(25.4 \%)$ presented with gastrointestinal symptoms, and $9(15.3 \%)$ had their feces tested positive for viral RNA; $38.5 \%$ and $8.7 \%$ of those with and without diarrhea, respectively $(\mathrm{p}=0.019)$, had stool viral RNA detected. In the meta-analysis, which included 60 studies and 4,243 patients, the prevalence of patients with gastrointestinal symptoms was $17.6 \%$; in that, $16.1 \%$ in studies from China, and $33.4 \%$ from other countries (South Korea, Singapore, Vietnam, United States, and United Kingdom). The gastrointestinal symptoms evaluated were anorexia $(26.8 \%)$, diarrhea $(12.5 \%)$, nausea/vomiting (10.2\%), and abdominal pain $(9.2 \%)$. The prevalence of RNA of the virus in feces was $48.1 \%$. Of these samples, $70.3 \%$ 
tested positive in the feces and could persist for more than 33 days from the onset of symptoms, even after the respiratory system had resulted negative for viral RNA. This meta-analysis also showed the prevalence of severe disease was more common in patients with gastrointestinal symptoms than in those without (17.1\% versus $11.8 \%$ ). The study limitations are underreporting of gastrointestinal symptoms and fewer studies outside China. Thus, healthcare professionals should pay attention to stool collection and procedures, such as endoscopy in patients with COVID-19.(27)

The systematic review and meta-analysis with 31 articles and 46,959 patients from China, by Cao et al., described the prevalence of symptoms such as fever $(87.3 \%)$, dry cough $(58.1 \%)$, dyspnea $(38.2 \%)$, myalgia or fatigue $(35.5 \%)$, chest pain $(31.2 \%)$, productive cough $(29.4 \%)$, sore throat $(12 \%)$, headache $(9.4 \%)$, and diarrhea $(6.8 \%)$. The incidence of ICU use, SARS, multiple organ failure, and mortality was $29.3 \%, 28.8 \%$, $8.5 \%$, and $6.8 \%$, respectively. The limitations of these studies were no foreigners included and no comments on viral presence in the feces. ${ }^{(28)}$

The meta-analysis made by Li et al., with 10 articles and 1,994 patients from China, showed similar results. The main symptoms reported were fever $(88.5 \%)$, cough $(68.6 \%)$, myalgia or fatigue $(35.8 \%)$, productive cough $(28.2 \%)$, and dyspnea (21.9\%). Other symptoms were headache or dizziness $(12.1 \%)$, diarrhea $(4.8 \%)$, and nausea and vomiting (3.9\%). Lymphopenia (64.5\%), increased C-reactive protein $(44.3 \%)$, increased lactate dehydrogenase $(28.3 \%)$, and leukopenia $(29.4 \%)$ were also recorded. In addition, men were more affected $(60 \%)$, and the fatality rate was $5 \%$. This study presented limitations by the number and quality of studies included. ${ }^{(29)}$

Sultan et al., performed a systematic review and meta-analysis of 47 studies with 10,890 patients. The prevalence of diarrhea was $7.7 \%$, nausea and vomiting $7.8 \%$, and abdominal pain $2.7 \%$. When comparing data from China and other countries, diarrhea $(5.8 \%$ versus $18.3 \%)$, nausea and vomiting (5.2\% versus $14.9 \%$ ), abdominal pain ( $2.7 \%$ versus $5.3 \%$ ), and liver abnormalities were more prevalent outside China. The liver alterations studied were AST elevation (total: 15.0\%; China: 14.9\%; other countries: 20.0\%), ALT (total: $15.0 \%$; China: $14.9 \%$; other countries: $19.0 \%$ ), and bilirubin (total or China: 16.7\%). There was no deeper analysis about the presence of viral RNA in the feces of these patients, but the authors found a variable prevalence of 15/50 (30\%), 7/10 (70\%), and $44 / 153(29 \%)$. The authors also provided a manual of recommendations for good practices in the management of COVID-19 with seven items; the main points are to obtain a detailed history of symptoms (typical and GIT), to verify liver function values upon admission of patients and monitor during hospitalization, and to evaluate the adverse reactions of drugs used in treatment. Regarding limitations, the patients were hospitalized (prevalence may be under or overestimated), the symptoms may have been analyzed inconsistently, information from laboratory tests was lacking, and there was no information about gastrointestinal symptoms being systematically evaluated at admission, among others. They concluded hepatic enzyme monitoring may be beneficial, and there is still no evidence to support the viral PCR test in the stools as a diagnosis or for monitoring of COVID-19 as a routine in clinical practice, because the results of other meta-analyses are conflicting and further studies on the subject are needed. ${ }^{(8)}$

\section{DISCUSSION}

With the evaluation of all these studies, one can see that there is still a lot of variation in the results regarding several points, and the factors studied were very different.

The mean age of patients ranged from 45.1 to 58.5 years, but only one article significantly correlated advanced age with gastrointestinal symptoms. ${ }^{(21)}$ In another article, advanced age was related to severity of the disease, but without assessing whether these patients had gastrointestinal symptoms. ${ }^{(25)}$ In general, all articles found more men affected with COVID-19 than women, and only one found a significant difference between non-medical women and more gastrointestinal symptoms. ${ }^{(26)}$ These results suggested that advanced age and male gender are risk factors for infection. The ACE2 receptor gene, target of SARV-CoV-2, is located on the $\mathrm{X}$ chromosome. A study still under review found that ACE2 expression increases in females and with sex hormones (which decrease with ageing); additionally, inflammatory cytokines decrease this expression (which increase with ageing and chronic diseases). This is contrary to what is currently thought, that is, increased ACE2 is to be blamed for the worse prognosis of COVID-19. SARS-CoV-2 binds directly to ACE2, causing downregulation of this receptor, which is even less in males, for example, and may explain the worse prognosis and the more prevalent infection in them. ${ }^{(31)}$

Regarding the comorbidities of infected patients, the most often cited were hypertension, diabetes mellitus, cerebrovascular disease, cardiovascular disease, lung 
disease, kidney disease, liver disease, and cancer, among others. However, only three articles reported a significant relation between gastrointestinal symptoms and comorbidities, and one of them ins chronic liver disease. ${ }^{(18,21,25)}$ On the other hand, the relation between COVID-19 and comorbidities also proved to be diverse. Very frequent comorbidities in the population were addressed and may have important significance in SARS-CoV-2 infection and prognosis.

The typical symptoms most often described are common in respiratory tract infections, such as fever (76.7\% to $94.9 \%)$, dry cough (38.6\% to $77.7 \%)$, productive cough $(14.2 \%$ to $43.0 \%)$, fatigue $(16.6 \%$ to $75.0 \%)$, dyspnea $(3.3 \%$ to $60.1 \%)$, headache $(8.0 \%$ to $21.6 \%)$, and sore throat $(8.11 \%$ to $17.0 \%){ }^{(8,18-29)}$ Other symptoms, such as anosmia and ageusia, are closely related to COVID-19, but only one study identified their presence ( $10.1 \%$ and $7.6 \%$, respectively). This study found a correlation between the presence of fatigue, myalgia, dyspnea, sore throat, anosmia, and ageusia with gastrointestinal symptoms. ${ }^{(22)}$ Two other studies also identified relation between gastrointestinal symptoms and typical symptoms; one with fever above $38.5^{\circ} \mathrm{C}$, fatigue, dyspnea, and headache, and the other with fatigue, sore throat, and dizziness. ${ }^{(18,26)}$

Regarding gastrointestinal symptoms, the prevalence showed great variation, ranging between $6.8 \%$ and $61.3 \%$ in these studies. This shows that there may be a regional trend in them, and studies with many patients from several countries are warranted to improve this relation between GIT and COVID-19. It is important to remember that two meta-analyses analysed China and other countries, and showed that the other sites presented with higher rates of gastrointestinal symptoms. ${ }^{(8,27)}$ However, there were few other countries, and many were in Asia. Nevertheless, this shows that gastrointestinal symptoms are important in this disease, may have a great impact in some countries, and should be evaluated based on history of suspected patients. The most often cited gastrointestinal symptoms were diarrhea $(8.14 \%$ to $33.7 \%)$, nausea/vomiting $(1.53 \%$ to $26.4 \%)$, anorexia (12.1\% to $40.0 \%)$, and abdominal pain $(0 \%$ to $14.5 \%) .{ }^{(8,18-29)}$

Several studies point out relations between laboratory changes and gastrointestinal symptoms or severity of the disease. Liver injury (elevation of AST, ALT, and bilirubin) was the most often evaluated, but elevations of C-reactive protein, procalcitonin, lactate dehydrogenase, D-dimer, and prothrombin were also verified, as well as a decrease in serum sodium levels, leukocytes, lymphocytes, and monocytes. ${ }^{(8,18,20,24,25,29)}$
Hence, it is very important to evaluate these parameters in patients hospitalized with COVID-19, since they may indicate a worse prognosis of the disease.

An important factor infrequently evaluated among the studies was the presence of viral RNA in stool samples from patients infected with SARS-CoV-2. This data would help us to understand if there is a possibility of fecal-oral transmission in this disease. Only four studies performed this analysis, in which two were meta-analyses and two retrospective studies. This test was positive in $0 \%$ to $48.1 \%$ of cases, with great heterogeneity in the results, but patients showing gastrointestinal symptoms presented more frequently the presence of virus RNA in feces. ${ }^{(8,18,19,27)}$ Some also identified that this result remained positive even after the negativity of respiratory tract samples. In one of them, $53.42 \%$ of feces samples were positive for the virus, and $23.29 \%$ were still positive when the respiratory tract was negative. ${ }^{(30)}$ In another, $70.3 \%$ of patients who had feces positive for the virus $(48.1 \%)$ also remained positive after respiratory clearance. ${ }^{(27)}$ However, studies did not evaluate whether the RNA present in feces represents infecting virus or only viral residues, with no capacity to infect. These data lead us to doubt whether the PCR test in feces of patients with COVID-19 should be done routinely or not. Therefore, further studies should be concerned and evaluate this issue, as possible fecal-oral transmission can have a major impact in developing countries that lack basic sanitation in many places.

Finally, the need for ICU admission ranged from $2.08 \%$ to $31.0 \%$, and cases that had evolved to death from $0 \%$ to $17.65 \%$. Several studies have commented on this, but only two presented with a significant difference between the presence of gastrointestinal symptoms and the need for ICU, or death. ${ }^{(8,21)}$ Thus, the investigation of these patients with gastrointestinal symptoms since the onset of the infectious condition becomes very important, considering many of them, more than those without these symptoms, may develop a more serious disease and need ICU, or even progress to death.

The articles in this review have several limitations. In general, the evaluation of the symptoms was done differently among them, there may be underreporting of gastrointestinal symptoms, and few were carried out with patients outside China. Additionally, the design was retrospective and had small samples, many did not evaluate the presence of viral RNA in feces, did not include asymptomatic patients, the data were hospital data, and the follow-up of patients was short. Moreover, because this review includes different study designs, 
there is a risk that the meta-analyses and systematic reviews also contain some retrospective article and may have duplicate results. The various study designs, per se, are also a limitation of this review, since the ideal would be to use the same model.

\section{COMMENTS}

The high rates of SARS-CoV-2 infection worldwide have caused the World Health Organization to declare a pandemic in 2020. Therefore, information about this new disease is of extreme importance, both in terms of pathophysiology and prevalence, severity, risk factors, diagnosis, and treatment. With this effort, we realize that the gastrointestinal tract can be greatly influenced by the disease, causing specific symptoms (diarrhea, abdominal pain, nausea, and vomiting) and laboratory changes (mainly of hepatic enzymes). Health professionals should pay attention to this, keeping alert to the modifications that can help in the diagnosis, and initiate early treatment in order to avoid bad prognosis. However, the results of prevalence of the gastrointestinal symptoms were very different, presenting a variation from $6.8 \%$ to $61.3 \%$. These symptoms were diarrhea ( $8.14 \%$ to $33.7 \%)$, nausea/vomiting ( $1.53 \%$ to $26.4 \%)$, anorexia $(12.1 \%$ to $40.0 \%)$, and abdominal pain $(0 \%$ to $14.5 \%)$. This important variation demands larger studies, with more patients and from various countries. As to the possibility of fecal-oral transmission, the presence of viral RNA was tested little, but was positive between $0 \%$ and $48.1 \%$. These data also varied a lot, preventing any conclusion about them, but they should also be studied further so that preventive measures can be taken. The RNA of the virus in feces does not necessarily indicate that there is fecal-oral transmission, and its evaluation in new studies to know if this presence is infectious or not is very important.

\section{AUTHORS' INFORMATION}

Almeida JF: http://orcid.org/0000-0003-3619-3003

Chehter EZ: http://orcid.org/0000-0002-2409-5871

\section{REFERENCES}

1. Zhou P, Yang XL, Wang XG, Hu B, Zhang L, Zhang W, et al. A pneumonia outbreak associated with a new coronavirus of probable bat origin. Nature. 2020;579(7798):270-3.

2. World Health Organization (WHO). WHO Coronavirus Disease (COVID-19) Dashboard [Internet]. Geneva: World Health Organization (WHO); 2020 [cited 2020 May 31]. Available from: https://covid19.who.int/
3. Corman VM, Muth D, Niemeyer D, Drosten C. Hosts and Sources of Endemic Human Coronaviruses. Adv Virus Res. 2018;100:163-88. Review.

4. Andersen KG, Rambaut A, Lipkin WI, Holmes EC, Garry RF. The proximal origin of SARS-CoV-2. Nat Med. 2020;26(4):450-5.

5. Eder P, Łodyga M, Dobrowolska A, Rydzewska G, Kamhieh-Milz J. Addressing multiple gastroenterological aspects of COVID-19. Pol Arch Intern Med. 2020;130(5):420-30. Review.

6. Xiao F, Tang M, Zheng X, Liu Y, Li X, Shan H. Evidence for gastrointestina infection of SARS-CoV-2. Gastroenterology. 2020;158(6):1831-3. e3.

7. Ding S, Liang TJ. Is SARS-CoV-2 Also an enteric pathogen with potential fecaloral transmission: a covid-19 virological and clinical review. Gastroenterology. 2020;159153-61.

8. Sultan S, Altayar O, Siddique SM, Davitkov P, Feuerstein JD, Lim JK, FalckYtter Y, El-Serag HB; AGA Institute. Electronic address: ewilson@gastro.org. AGA Institute rapid review of the gastrointestinal and liver manifestations of covid-19, meta-analysis of international data, and recommendations for the consultative management of patients with COVID-19. Gastroenterology. 2020;159(1):320-34. e27.

9. Monkemuller K, Fry L, Rickes S. Covid-19, coronavirus, SARS-CoV-2 and the small bowel. Rev Esp Enferm Dig. 2020;112(4):383-8. Review.

10. Platt AM, Mowat AMI. Mucosal macrophages and the regulation of immune responses in the intestine. Immunol Lett. 2008;119(1-2):22-31. Review.

11. Ziegler CGK, Allon SJ, Nyquist SK, Mbano IM, Miao VN, Tzouanas CN, Cao Y, Yousif AS, Bals J, Hauser BM, Feldman J, Muus C, Wadsworth MH 2nd Kazer SW, Hughes TK, Doran B, Gatter GJ, Vukovic M, Taliaferro F, Mead BE, Guo Z, Wang JP, Gras D, Plaisant M, Ansari M, Angelidis I, Adler H, Sucre JMS, Taylor CJ, Lin B, Waghray A, Mitsialis V, Dwyer DF, Buchheit KM, Boyce JA, Barrett NA, Laidlaw TM, Carroll SL, Colonna L, Tkachev V, Peterson CW, Yu A, Zheng HB, Gideon HP, Winchell CG, Lin PL, Bingle CD, Snapper SB, Kropski JA, Theis FJ, Schiller HB, Zaragosi LE, Barbry P, Leslie A, Kiem HP, Flynn JL, Fortune SM, Berger B, Finberg RW, Kean LS, Garber M, Schmidt AG, Lingwood D, Shalek AK, Ordovas-Montanes J; HCA Lung Biological Network. Electronic address: lung-network@humancellatlas.org; HCA Lung Biological Network. SARS-CoV-2 receptor ACE2 is an interferon-stimulated gene in human airway epithelial cells and is detected in specific cell subsets across tissues. Cell. 2020;181(5):1016-35. e19.

12. Leung JM, Yang CX, Tam A, Shaipanich T, Hackett TL, Singhera GK, et al. ACE-2 expression in the small airway epithelia of smokers and COPD Patients: Implications for COVID-19. Eur Respir J. 2020;55(5):2000688.

13. Hussain A, Bhowmik B, do Vale Moreira NC. COVID-19 and diabetes: Knowledge in progress. Diabetes Res Clin Pract. 2020;162:108142.

14. Fosbøl EL, Butt JH, Østergaard L, Andersson C, Selmer C, Kragholm K, et al. Association of angiotensin-converting enzyme inhibitor or angiotensin receptor blocker use with covid-19 diagnosis and mortality. JAMA. 2020; 324(2):168-77.

15. Li J, Wang X, Chen J, Zhang H, Deng A. Association of renin-angiotensin system inhibitors with severity or risk of death in patients with hypertension hospitalized for coronavirus disease 2019 (COVID-19) infection in Wuhan, China. JAMA Cardiol. 2020;5(7):825-30.

16. Vankadari N, Wilce JA. Emerging WuHan (COVID-19) coronavirus: glycan shield and structure prediction of spike glycoprotein and its interaction with human CD26. Emerg Microbes Infect. 2020;9(1):601-4.

17. Tai $W$, He L, Zhang $X, P u$ J, Voronin D, Jiang $S$, et al. Characterization of the receptor-binding domain (RBD) of 2019 novel coronavirus: implication for development of RBD protein as a viral attachment inhibitor and vaccine. Cell Mol Immunol. 2020;17:613-20.

18. Jin X, Lian JS, Hu JH, Gao J, Zheng L, Zhang YM, et al. Epidemiological, clinical and virological characteristics of 74 cases of coronavirus-infected disease 2019 (COVID-19) with gastrointestinal symptoms. Gut. 2020;69(6):1002-9.

19. Lin L, Jiang $X$, Zhang Z, Huang $S$, Zhang Z, Fang Z, et al. Gastrointestinal symptoms of 95 cases with SARS-CoV-2 infection. Gut. 2020;69:997-1001.

20. Pan L, Mu M, Yang P, Sun Y, Wang R, Yan J, et al. Clinical characteristics of COVID-19 patients with digestive symptoms in Hubei, China. Am J Gastroenterol. 2020;115(5)766-73. 
21. Wan Y, Li J, Shen L, Zou Y, Hou L, Zhu L, et al. Enteric involvement in hospitalised patients with COVID-19 outside Wuhan. Lancet Gastroenterol Hepatol. 2020;5(6):534-5.

22. Redd WD, Zhou JC, Hathorn KE, McCarty TR, Bazarbashi AN, Thompson CC, et al. Prevalence and characteristics of gastrointestinal symptoms in patients with SARS-CoV-2 infection in the United States: a multicenter cohort study. Gastroenterology. 2020;159(2):765-7. e2.

23. Nobel YR, Phipps M, Zucker J, Lebwohl B, Wang TC, Sobieszczyk ME, et al. Gastrointestinal symptoms and COVID-19: case-control study from the United States. Gastroenterology. 2020;159(1):373-5. e2.

24. Cholankeril G, Podboy A, Aivaliotis VI, Tarlow B, Pham EA, Spencer S, et al. High prevalence of concurrent gastrointestinal manifestations in patients with SARS-CoV-2: early experience from California. Gastroenterology. 2020;159(2):775-7.

25. Zhang JJ, Dong X, Cao YY, Yuan YD, Yang YB, Yan YQ, et al. Clinical characteristics of 140 patients infected with SARS-CoV-2 in Wuhan, China. Allergy. 2020;75(7):1730-41.
26. Zhou Z, Zhao N, Shu Y, Han S, Chen B, Shu X. Effect of gastrointestinal symptoms on patients infected with COVID-19. Gastroenterology. 2020; 158(8):2294-7.

27. Cheung KS, Hung IF, Chan PP, Lung KC, Tso E, Liu R, et al. Gastrointestinal manifestations of SARS-CoV-2 infection and virus load in fecal samples from the Hong Kong cohort and systematic review and meta-analysis. Gastroenterology. 2020;159(1):81-95.

28. Cao Y, Liu X, Xiong L, Cai K. Imaging and clinical features of patients with 2019 novel coronavirus SARS-CoV-2: a systematic review and meta-analysis. J Med Virol. 2020 Apr 3:10.1002/jmv.25822. Review.

29. Li L, Huang T, Wang Y, Wang Z, Liang Y, Huang T, et al. COVID-19 patients' clinical characteristics, discharge rate, and fatality rate of meta-analysis. $J$ Med Virol. 2020;92(6):577-83. Review.

30. Cipriano M, Ruberti E, Giacalone A. Gastrointestinal infection could be new focus for coronavirus diagnosis. Cureus. 2020;12(3)e7422.

31. Chen J, Jiang $\mathrm{Q}$, Xia X, Liu K, Yu Z, Tao W, et al. Individual variation of the SARS-CoV2 receptor ACE2 gene expression and regulation. Aging Cell. 2020; 19(7):e13168. 